\title{
Volume anormal de negociação de ações e demanda por informação: análise para ações de alta liquidez da Bovespa
}

\author{
Abnormal volume and demand for information: an analysis of Bovespa's high-liquid stocks
}

\section{Rodolfo T. da F. Nicolay ${ }^{\dagger, *^{*}}$, Patrícia Rodrigues Passos ${ }^{\S}$, Vanessa Cristina dos Santos ${ }^{\dagger}$ \\ Como citar esse artigo. Nicolay, R.T.F.; \\ Resumo} Passos, P.R.; dos Santos, V.C. Volume anormal de negociação de ações e demanda por informação: análise para ações de alta liquidez da Bovespa. Revista Mosaico, v.11, n.2, p. 76-86, 2020 .

Este estudo examina a relação entre o volume das pesquisas realizadas na Internet e o volume total negociado no mercado de ações. Os resultados demonstram que as buscas online, de um modo geral, captam a atenção do investidor e podem interferir no volume transacionado no mercado no curto prazo. O trabalho também analisa alterações no volume transacionado quando o preço corrente das ações está acima ou abaixo dos preços anteriores das últimas 52 semanas. Para o trabalho foi utilizada uma carteira teórica formada pelas principais ações do IBOVESPA, principal benchmark do desempenho das ações da bolsa de valores brasileira - $[\mathrm{B}]^{3}$ (Brasil, Bolsa, Balcão) - cujo objetivo é o de ser refletir as ações com maior volume negociado. Em ambas as análises é possível identificar que a atenção dispendida para a ação nas buscas na internet está relacionada com o volume anormal de negociação das ações da bolsa, corroborando a hipótese de racionalidade limitada dos agentes.

Nota da Editora. Os artigos publicados na Revista Mosaico são de responsabilidade de seus autores. As informações neles contidas, bem como as opiniões emitidas, não representam pontos de vista da Universidade de Vassouras ou de suas Revistas.

Palavras-chave: Volume anormal, Mercado de ações, Google Trends, Bovespa.

\begin{abstract}
This study examines the relation between the volume of Internet searches and the total volume trading in the stock market. The results demonstrate that online searches, in general, capture the attention of the investor and may interfere with the volume traded in the market in the short term. The work also analyzes whether changes in traded volume occur when the current stock price is above (below) the previous 52-week prices. For this paper was formed a theoretical portfolio with the main shares of the BOVESPA INDEX, principal benchmark of the Brazilian stock exchange - $[\mathrm{B}]^{3}$ (Brasil, Bolsa, Balcão) - whose objective is to reflect the shares with high volume traded. In both analyzes, the observed effects are consistent with the presence of behavioral aspects in the origin of the abnormal volume traded for liquid stocks in the Brazilian stock market.
\end{abstract}

Keywords: Abnormal volume, stock market, Google Trends, Bovespa.

\section{Introdução}

Nos estudos sobre as origens e os efeitos dos comportamentos observados na sociedade atual, cada vez mais globalizada, onde o significado de contemporaneidade adquire novos sentidos, não se discute a importância da Internet. Juntamente com esse novo status quo, cresce o número de pesquisas associando a relação entre a Internet e o surgimento de novos hábitos.

Da et al. (2011) e Mondria, Wu e Zhang (2010) foram pioneiros em usar o volume de pesquisas na Internet em economia financeira. Ao incluir o resultado dessas pesquisas no âmbito das finanças comportamentais, buscam quantificar como essas buscas estão relacionadas com os interesses dos investidores e quais seriam suas implicações nos movimentos observáveis de mercado. As buscas realizadas nesse ambiente têm sido utilizadas como variável explicativa do volume de transações realizadas. O presente trabalho se baseia nos estudos realizados por Joseph, Wintoki e Zhang (2011), ao analisar a relação entre as buscas online no Google e alterações do volume negociado no mercado acionário brasileiro. Seguindo Huddart, Lang e Yetman (2005), também se analisa a relação entre preços passados e o volume negociado, quando uma ação ultrapassa determinado nível de preço para um intervalo anterior de 52 semanas (benchmark), em particular, para as ações com histórico de alta liquidez na bolsa.

Um dos principais objetivos das finanças comportamentais é o de compreender como as implicações sistemáticas do mercado interferem nos

Afiliação dos autores:

†Universidade Católica de Petrópolis, Petrópolis, RJ, Brasil

Universidade Candido Mendes, Rio de Janeiro, RJ, Brasil

${ }^{\S}$ Banco do Brasil, Rio de Janeiro, RJ, Brasil

* Email de correspondência: r-nicolay@hotmail.com 
traços psicológicos dos agentes (STRACCA, 2004). Se sob a ótica da abordagem convencional, os agentes possuem expectativas racionais, não cometem erros sistemáticos e conhecem a "verdadeira" probabilidade da distribuição, as teorias comportamentais apresentam um indivíduo não tão racional, com esquemas internos de funcionamento baseados em crenças originadas em experiências anteriores (boas ou ruins). A aversão à perda, proposta por Kahneman e Tverski (1979), baseiase na constatação de que as pessoas sentem muito mais a dor da perda do que o prazer obtido com um ganho equivalente. Na teoria da dissonância cognitiva, proposta por Festinger (RIECKEN; SCHACHTER, 1956), quando dois pensamentos simultâneos são inconsistentes, produz-se um estado de dissonância cognitiva, tratada como uma experiência desagradável pelo indivíduo, que se esforçará para reduzi-lá a partir da mudança das crenças internas.

As finanças comportamentais ainda não se encontram em um estágio de amadurecimento que invalide os preceitos das abordagens normativas, como a Teoria da Utilidade (VON NEUMANN; MORGENSTERN, 1947), ou da Hipótese de Mercado Eficiente (HME), (FAMA; 1970). Mas trata-se de abordagem alternativa que se propõe a elucidar a origem dos movimentos de mercado (anomalias) observados empiricamente. Com a inclusão de aspectos comportamentais em finanças, amplia-se as discussões sobre as idiossincrasias observadas no mercado e a análise de suas origens.

Ferris et al. (1988) analisaram como o volume negociado pode ser afetado pelas abordagens da taxloss-selling hypothesis" ${ }^{1}$ e da teoria da "Disposition Effect" (SHEFRIN E STATMAN; 1985), ou seja, como os preços passados, para diferentes níveis de preços de ações, interferem no volume transacionado. Para Camerer e Lovallo (1999), o excesso de confiança dos investidores, o que os leva a concluir que suas estratégias são mais eficientes do que as dos demais, inclui habilidades individuais (variável psicológica) nas tomadas de decisões nos negócios. Trata-se de incluir experiências (positivas) passadas no comportamento mental, corroborando o trabalho realizado por Huddart, Lang e Yetman (2005), quando analisaram se o histórico dos preços das ações influencia na tomada de decisão em investir.

O presente trabalho tem como objetivo principal analisar a relação entre as buscas online no Google e alterações no volume negociado para ações de alta liquidez do mercado acionário brasileiro. Em específico, o trabalho objetiva: (i) analisar se as buscas realizadas na Internet são variável explicativa do incremento do volume negociado observado no mercado, (ii) observar se o incremento constatado, para o curto prazo, resulta em um volume anormal de transações de compra e/ ou venda de ações. Ademais, o trabalho inclui como variável explicativa dos movimentos de mercado doméstico a Operação Lava Jato e seus efeitos sobre o volume total negociado. Como variáveis de controle, de modo a dar mais robustez ao modelo proposto, foram incluídas o retorno das ações, a volatilidade e o anúncio de dividendos, com o objetivo de dirimir uma interpretação equivocada dos coeficientes das variáveis explicativas.

O método utilizado foi uma regressão simples pelo Método dos Mínimos Quadrados Ordinários (MQO). Os resultados das estimações, com dados em painéis, foram significativos para explicar o volume anormal, onde conclui-se que as variáveis utilizadas foram capazes de demonstar a relação entre o volume das pesquisas na Internet e o volume negociado (JOSEPH, WINTOKI E ZHANG; 2011) e que o investidor direciona sua atenção aos extremos em relação a um histórico de preços para uma janela de 52 semanas (HUDDART, LANG E YETMAN; 2005).

\section{Revisão da Literatura}

\section{Teoria das finanças: dos mercados eficientes às finanças comportamentais}

Sob a ótica da abordagem tradicional, as tomadas de decisão dos agentes econômicos objetivam a maximização da riqueza e são baseadas em informações fidedignas divulgadas pelas empresas e pelo mercado. Isto implica dizer que a preferência individual é estática com comportamento adverso ao risco e que o preço dos ativos somente são influenciados com base nos fundamentos de mercado (TUYON; AHMAD, 2016), cujos preços das ações apresentam um comportamento randômico (BACHELIER, 1900) e um modelo martingal (SAMUELSON,1965).

Como resultado, os seguintes comportamentos se tornariam observáveis no mercado: (i) não previsibilidade dos preços das ações, o que impediria as situações de arbitragem; e (ii) mantidos os comportamentos dos investidores e dos preços como atribuídos, um processo aleatório de séries financeiras com distribuição independente e idêntica, como um Gaussiano (distribuição normal) com média zero e variância constante (LIM; LIEW; WONG; 2005).

Guiado por essas teorias, Fama (1965); 1970) idealizam a Hipótese de Mercado Eficiente (HME), onde apresenta um quadro teórico para o comportamento do mercado a partir da disponibilidade das informações, de modo que os preços se modificam imediatamente diante de uma nova informação divulgada, e onde os agentes econômicos são totalmente racionais. Fama (1965; 1970) apresenta três níveis de equilíbrio na HPE: fraco, 
semiforte e forte. No primeiro nível, o preço dos ativos é reflexo das informações publicamente disponíveis e do histórico de seus preços. Para o segundo, o preço dos ativos é o reflexo tanto das informações passadas quanto das atuais, exigindo maior embasamento teórico por parte dos investidores para analisar qual é o reflexo imediato das novas informações no preço das ações. No mercado eficiente forte, os preços também refletem, adicionalmente e imediatamente, as informações não disponíveis (ocultas ou privilegiadas).

Como consequência da teoria proposta por Fama $(1965 ; 1970)$, os aspectos psicológicos dos agentes não interferem no funcionamento do mercado e suas expectativas são exclusivamente racionais, com ausência de erros sistemáticos e com o conhecimento da verdadeira probabilidade da distribuição. Isso implica dizer que, na presença de anomalias, onde as transações não se baseiam em informações consistentes sobre a expectativa futura dos preços, tanto os fundamentos quanto as crenças do mercado influenciam no preço do ativo, de modo a corrigir estas distorções (BLANCHARD; 1979).

A importância da expectativa dos preços futuros de um ativo sobre os seus preços atuais foi enfatizada, em especial, na literatura sobre a incerteza "extrínseca" ou "endógena" (CASS; SHELL; 1983), em oposição à incerteza "intrínseca" ou "exógena" que está relacionada com os fundamentos futuros. A teoria das crenças racionais (KURZ, 1994; KURZ; MOTOLESE, 2001) tem se concentrado na incerteza endógena e nas expectativas de auto justificação como principal fonte de incerteza no mercado.

As situações acima, representativas de anomalias de mercado, são definidas como características observáveis (empíricas) do comportamento dos investidores, as quais não podem ser explicadas pelo modelo da utilidade esperada (STRACCA, 2004). Sob essa perspectiva, a abordagem comportamental em finanças propõe-se à investigação de quais são os vieses comportamentais, inclusive crenças individuais (heurísticas), mais importantes e sistemáticos que caracterizam os agentes econômicos. Assim, rejeita-se a visão baseada somente na maximização da utilidade esperada quando da tomada de decisão em finanças. $\mathrm{Na}$ origem dessa rejeição está a evidência de que os agentes, tanto em experimentos controlados como em situações da vida real, se comportam de uma maneira que viola os axiomas da utilidade esperada (STARMER, 2000).

Segundo Stracca (2004), o foco das finanças comportamentais é uma descrição positiva do comportamento humano, especialmente sob risco e incerteza, em vez de uma análise normativa do comportamento, que é mais típica da abordagem convencional. Trata-se de compreender como as características psicológicas dos agentes econômicos influenciam, de modo sistemático, o mercado financeiro.

\section{Finanças comportamentais e Volume Anormal de açõs negociadas ${ }^{3}$}

A pesquisa em aprendizagem e memória sugere que os indivíduos provavelmente se concentrarão em observações extremas, mesmo que não esclarecedoras e irrelevantes (ver, por exemplo, FREDERICKSON E KAHNEMAN; 1993, FISKE E TAYLOR; 1991). Ampliando essa perspectiva à área de finanças, muitos dos estudos realizados avaliam a atenção do investidor para os extremos em relação a um histórico de preços para uma janela de 52 semanas. Refere-se ao intervalo onde as estatísticas sobre o desempenho das ações são comumente divulgadas e consideradas nas análises técnicas para definição dos níveis de preço de resistência e de suporte, usualmente, divulgadas em sites especializados da área.

$\mathrm{Ou}$ seja, os efeitos da racionalidade limitada sugerem que o aumento no volume transacionado está associado ao deslocamento ou com o aumento da atenção do investidor para determinada ação quando o seu preço se move para fora de determinado intervalo de preços. Esse resultado é mais evidente quanto maior for o tempo desde que o período de avaliação (benchmark) foi estabelecido, com a diminuição gradual da atenção dispensada. Para Huddart, Lang e Yetman (2005), esse efeito pode ser mais acentuado para as operações de compra, uma vez que os investidores individuais têm uma grande variedade de potenciais ações para adquirir, mas estão limitados em suas opções de vendas às empresas que fazem parte de seus portfólios.

Pode-se dizer que a atenção dada pelo investidor às informações disponibilizadas está presente, de modo geral, em ambas as abordagens (tradicional e comportamental). O que se discute, portanto, é se o investidor inicia suas operações a partir da informação (passada) dos preços mínimos e máximos ocorridos em determinada série histórica ou se ele decide a partir da constatação de que os preços atuais já refletem toda a informação disponível e, assim, seriam os preços "justos". É essa decisão que reflete em um maior ou menor número de transações no mercado e, consequentemente, em oscilações do volume financeiro transacionado, já que este último é o resultado do preço atual da ação multiplicado pela quantidade negociada.

Sob a perspectiva apresentada por Fama (1970), se os mercados são eficientes, com investidores homogêneos, os preços refletem todas as informações sobre o ativo, com os ajustes de mercado se refletindo em mudanças de preços, mas não necessariamente no número de transações. De acordo com Van der Sar (2004), qualquer alteração no nível dos seus preços representa uma revisão das expectativas a partir da inclusão do 
efeito de novas informações. Assim, caso persistam movimentos no mercado de euforia (desânimo), eles podem ser reflexo de uma reação tardia às notícias, resultando em distorções no mercado, seguidas de uma posterior correção.

Ou seja, na presença de qualquer comportamento irracional, há um movimento de correção iniciada pelo mercado(investidores racionais) no sentido do equilíbrio. No entanto, conforme assinalado por Van der Sar (2004), como os (altos) valores financeiros envolvidos podem resultar em um distanciamento entre os preços praticados e seus valores intrínsecos, a correção esperada pode ser afetada quando: (i) os recursos financeiros de arbitragem são limitados em quantidade e duração; (ii) há imprevisibiliade no comportamento dos investidores irracionais; e (iii) os investidores racionais podem ter aversão ao risco e serem ávidos em consumir.

Statman e Thorley (1999) discutem que os determinantes do volume negociado são mal-entendidos e os modelos de agentes econômicos racionais que maximizam a utilidade não se encaixam bem nos padrões observados, mas os modelos comportamentais dão novas previsões testáveis sobre os determinantes do volume.

Os experimentos realizados por Kahneman e Tversky (1979) demonstraram que, mesmo ciente das probabilidades envolvidas, a percepção de risco dos indivíduos muda a partir do modo como as perspectivas são apresentadas e dos valores monetários envolvidos. Mais uma vez, trata-se de uma constatação empírica que questiona a validade da Teoria da Utilidade (VON NEUMANN; MORGENSTERN, 1947), quando sustenta que as decisões dos investidores são baseadas somente em probabilidades conhecidas e na maximização da utilidade.

\section{Ferramentas de Informação Digital e Volume Anormal}

A atenção é um recurso cognitivo escasso (KAHNEMAN, 1973). Sob esse enfoque, deduz-se que há um direcionamento/foco para as informações mais relevantes para o indivíduo. Como contribuição à racionalização da atenção, atualmente, a Internet disponibiliza as principais ferramentas de pesquisa (sites de buscas, fóruns e blogs) que permitem aos seus usuários o direcionamento das consultas para um conjunto compilado de dados cada vez maior. A utilização desses recursos também é útil na obtenção de informações que podem ajudar os investidores em suas estratégias de investimentos. Bank, Larch e Peter (2011) encontraram uma relação entre o aumento no volume de busca na Internet e na atividade de negociação e da liquidez das ações, resultando em retornos futuros elevados no curto prazo.
Wysocki (1998) foi o primeiro a demonstrar que os fóruns postados no Yahoo podem prever mudanças no volume de transações e nos retornos de títulos no dia seguinte ao da postagem. Da et al. (2011) usaram a frequência de busca no Google como uma medida direta da atenção do investidor e consideram que esse proxy pode antecipar os preços das ações nas próximas duas semanas. Eles demonstram que o volume de busca na Internet mede a atenção mais oportuna quando comparada com outras variáveis de atenção mais consolidadas e, principalmente, capta a atenção do investidor individual.

As redes sociais, como Twitter e Facebook, também podem fornecer informações sobre as pesquisas realizadas por seus usuários (JOSEPH; WINTOKI; ZHANG, 2011). Rubin e Rubin (2010) analisaram a frequência de edição da Wikipédia para medir a variação de uma cross section no processamento de informações para 30 empresas do Índice Dow Jones. Eles relatam evidências de que uma maior frequência de edição da ferramenta está associada a erros menores de previsão de analistas e uma menor dispersão de previsão.

Observa-se cada vez mais a utilização de ferramentas sobre as buscas realizadas pelos indivíduos nos diferentes meios digitais por parte dos pesquisadores em finanças comportamentais. Uma ferramenta utilizada com esta finalidade é o Google Trends, que fornece dados sobre a frequência dos termos de pesquisa dos usuários no Google desde janeiro de 2004. Como plataforma de busca amplamente utilizada, é provável que seja representativa do comportamento de busca na Internet da população (DA et al., 2011).

\section{Metodologia}

O trabalho analisa o impacto da atenção do investidor sobre o volume anormal de negociação de ações. A análise é feita por meio de uma estratégia empírica utilizando uma estrutura de dados em painel, considerando as empresas com maior liquidez na bolsa.

\section{Base de dados}

O estudo focou no mercado de capitais brasileiro, mais especificamente no volume negociado das ações de alta liquidez na bolsa de valores - $[\mathrm{B}]^{3}$ (Bolsa). A partir da composição do Índice BOVESPA(IBOVESPA) ${ }^{5}$, cuja meta é refletir as ações com maior volume negociado no mercado acionário brasileiro, foi criada uma carteira teórica usando os critérios:

a) Obtenção das carteiras teóricas IBOVESPA de janeiro/2010 a dezembro/2016 .

b) Seleção das ações com, pelo menos, $2 \%{ }^{7}$ de participação em cada uma das carteiras e que estiveram 
presentes em todo o intervalo da pesquisa.

c) Criação de uma carteira teórica anual de ações que atendam aos critérios acima.

d) Mantidas ações BBAS3, mesmo tendo participação abaixo da meta nos períodos: 2010 (maioagosto); 2015 (setembro-dezembro); e 2016 (janeiroabril).

e) Ao final, foram obtidas 7 carteiras teóricas do IBOVESPA, uma para cada ano, com 9 ações (Preferencias - PN e Ordinárias - ON) para 7 empresas distintas: Brasil, Bolsa e Balcão - $[\mathrm{B}]^{3}(\mathrm{BVMF} 3)^{8}$, Banco do Brasil S.A. (BBAS3), Banco Bradesco S.A. (BBDC4), ITAUSA S.A. (ITSA4), ItauUnibanco S.A. (ITUB4), Petróleo Brasileiro S.A. - PETROBRAS (PETR3 e PETR4) e Vale S.A. (VALE3 e VALE4).

Construiu-se uma série histórica dos preços passados das principais ações do IBOVESPA, com ênfase nos seus máximos e mínimos, para determinar se as negociações ocorrem com mais frequência quando os preços estão fora desses limites (HUDDART; LANG; YETMAN, 2005). Os preços de fechamento dos ativos foram coletados para períodos consecutivos de 52 semanas visando encontrar os máximos e mínimos 9 .

Similar a Huddart, Lang e Yetman (2005), os preços dos 20 dias (4 semanas) que precedem a semana de observação (semana $i$ ) foram excluídos, visando refletir a suposição de que o ponto de referência se ajusta gradualmente. Senão, negociações com base em preços extremos seriam mais raras, pois tal ponto seria reiniciado. A Figura 1 ilustra tais intervalos:

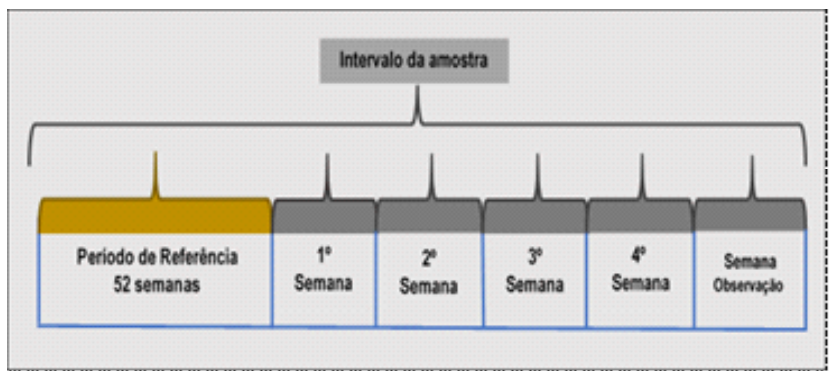

Figura 1. Intervalos da pesquisa do volume anormal.

Fonte: Elaborada pela autora, baseada em Borges (2014).

\section{Cálculo do Volume Anormal}

O volume semanal negociado a cada empresa é a variável dependente e o volume semanal total do mercado a explicativa, como estimativa para obter o volume anormal do mercado (VOLABN).

Para Huddart, Lang e Yetman (2005), a regresão inicial (1) do volume anormal é dada:

$$
\mathrm{VOL}_{\mathrm{it}}=\beta_{0}+\beta_{1} \mathrm{MVOL}_{\mathrm{t}}+\mathrm{e}_{\mathrm{it}}
$$

e, similar à Ferris et al. (1998), trata-se de regressão (2) com dois estágios:

$$
\mathrm{V}_{\mathrm{it}}=\beta_{\mathrm{i}}+\beta_{\mathrm{i}} \mathrm{V}_{\mathrm{im}}+\mathrm{e}_{\mathrm{it}}
$$

Para o primeiro estágio $\left(\mathrm{V}_{\mathrm{it}}\right)$, onde $\mathrm{V}_{\mathrm{it}}=$ volume para a ação $i$ no dia $t$ :

$V_{i t}=\frac{\text { Número de ações da empresa i negociadas no dia } \mathrm{t}}{\text { Total do número das ações da empresa i disponíveis no dia } \mathrm{t}^{\prime}}$

Para o segundo estágio $\left(\mathrm{V}_{\mathrm{im}}\right)$ :

$V_{i m}=\frac{\text { Número de ações de todas as empresas negociadas no dia t }}{\text { Total do número das ações da empresa i disponíveis no dia } \mathrm{t}^{\prime}}$

Onde $\mathrm{e}_{\mathrm{it}}=$ volume de negócios anormal (VOLABN) para a ação $i$ no dia $t$.

Retornando às regressões (1) e (2): $\mathrm{VOL}_{\mathrm{it}}$ é correspondente a $\mathrm{V}_{\mathrm{it}}$ e $\mathrm{MVOL}_{\mathrm{t}}$ é equivalente a $\mathrm{V}_{\mathrm{im}}$ e, em ambas as equações, o VOLABN é estimado pelo erro $\left(\mathrm{e}_{\mathrm{it}}\right)$.

Segundo Beaver (1968), Dyl (1977) e Lakonishok e Smidt (1986), e e é a fração das ações $i$ negociadas no dia $t$ após removidos os efeitos dos eventos ${ }^{10}$ do mercado. $\mathrm{VOL}_{\text {it }}$ é obtido pelo volume financeiro negociado para cada ação da amostra e MVOL o volume financeiro de ações negociadas. Estimado em duas etapas: multiplicar numerador e denominador em (3) e (4), para obter $\mathrm{VOL}_{\text {it }}$ e o do $\mathrm{MVOL}_{\mathrm{t}}$, estimando o erro (VOLABN) a partir da regressão (5):

$$
\mathrm{VOL}_{\mathrm{it}}=\beta_{0}+\beta_{1} \mathrm{MVOL}_{\mathrm{t}}+\epsilon_{\mathrm{it}}
$$

\section{Modelo econométrico}

As principais variáveis explicativas para o modelo econométrico são as variáveis Dummy de Máxima (MAX) e Mínima (MIN), que permitem identificar em quais semanas os preços de fechamento das ações de cada empresa está fora do range de negociação avaliado (HUDDART; LANG; YETMAN, 2005). Na semana de observação $(i)$, quando os preços correntes ${ }^{11}$ estiverem acima do preço máximo (mínimo) das últimas 52 semanas, a variável MAX (MIN) assume valor 1 e, caso contrário, o valor 0 .

Statman e Thorley (1999) sugerem que a relação entre volume e retornos pode ser assimétrica, com retornos negativos reduzindo o volume mais do que o seu incremento quando da presença de retornos positivos. Barber e Odean (2002) mostram que os investidores de varejo são mais propensos a negociar quando os retornos são grandes nos seus valores absolutos. Sob estas perspectivas, como variável de controle, foram incluídos na regressão os retornos contemporâneos, considerados aqueles da semana de observação, e os passados, ambos separados pelos seus sinais. Onde, $i$ 
$=1$ a 4 , com RET i sendo o retorno durante a semana - $i$, obtido através da razão entre o último preço e o primeiro preço de fechamento da semana anterior à de observação, excluindo os dividendos.

A inclusão da volatilidade (SDVOL) no modelo considerou os argumentos apresentados por Huddart, Lang e Yetman (2005): (i) o aumento da volatilidade do retorno pode refletir uma maior incerteza no mercado, o que pode levar a negociação adicional; e (ii) a Teoria da Perspectiva (KAHNEMAN; TVERSKY, 1979) sugere que uma maior volatilidade pode afetar as decisões de venda. Huddart, Lang e Yetman (2005), estabelecem a volatilidade como o desvio padrão anualizado dos retornos das ações calculado a partir das 26 observações semanais anteriores à semana de observação.

Para este estudo, diferentemente do proposto por Huddart, Lang e Yetman (2005), embora o cálculo da SDVOL também se inicie a partir das observações sugeridas, este se estende a intervalos consecutivos para abrangência de todo o intervalo da pesquisa. Difere, ainda, o fato de que a volatilidade é calculada com base na utilização das cotações de fechamento diárias de cada empresa para as semanas da amostra (Desvio Padrão da Amostra).

A introdução do anúncio do pagamento (futuro) de dividendos por parte das empresas como variável de controle visa conferir mais robustez ao modelo e se justifica pelos seguintes fatos: (i) o volume negociado é afetado quando da sua divulgação; (ii) trata-se de evento de comunicação obrigatória por parte da empresa e, consequentemente, aguardada pelo mercado; e (iii) alguns gestores incluem em suas carteiras empresas com bom histórico de pagamento de dividendos. Registrarse, no entanto, que a variável considera somente o anúncio e não o pagamento efetivo dos dividendos ${ }^{12}$. Ou seja, o anúncio do pagamento dos dividendos ${ }^{13}$, embora ainda não represente um fluxo de caixa efetivo para o investidor, está relacionado com uma expectativa futura de ganho e, consequentemente, pode afetar o volume negociado.

$\mathrm{O}$ anúncio de dividendos (EARNANN) foi incluída no modelo como uma variável Dummy, assumindo o valor 1, quando do anúncio, e 0 , caso contrário, nas semanas seguintes ao intervalo de 52 semanas.

A principal variável de interesse do trabalho, a frequência de busca no Google para o ticker de negociação da ação foi considerada como proxy para determinar alterações no volume negociado. Da et al. (2011) demonstram que o volume de busca na Internet capta a atenção do investidor individual e pode antecipar preços de ações nas próximas duas semanas. A escolha pelo ticker foi baseada nos estudos de Joseph, Wintoki e Zhang (2011) como medida de comportamento de pesquisa de investidores, considerando que os esforços nelas se concentram em uma decisão de investimento específica.

A frequência de busca pelos tickers das ações da amostra (BBAS3; BBDC4; BVMF3; ITSA4; ITUB4; PETR3; PETR5; VALE3; e VALE5) no Google ${ }^{14}$ foi obtida com o uso do Google Trends ${ }^{15}$, que disponibiliza o índice de volume de pesquisa (IVP) semanal, onde o número de pesquisas para determinado termo é escalado pela sua média de séries temporais. A seguir, os critérios utilizados na pesquisa do tickers das ações:

a) A Abrangência limitou-se ao Brasil;

b) Na procura pelos termos de pesquisa, optouse pelas opções: "Período Personalizado" e "Ano Completo" - para dados semanais;

c) O intervalo utilizado compreendeu o período de 02.01.2011 a 25.12.2016, resultando em uma amostra de 313 semanas; e

d) Nas buscas dos respectivos tickers foram usadas letras maiúsculas, ex. VALE3.

Para aumentar a velocidade de resposta, o Google Trends calcula o IVP a partir de um subconjunto aleatório dos dados de pesquisa históricos reais, como efeito, para um mesmo termo de pesquisa os resultados podem ser ligeiramente diferentes quando são consultados em diferentes momentos.

O Google Trends apresenta os dados semanais para um intervalo de domingo a sábado. Para o estudo em questão, como os dados também foram consolidados por semana, representativa de um dado da amostra, destaca-se que, diferentemente da Solução acima, a consolidação ocorreu com base nos dias úteis semanais, com início na segunda e término na sexta-feira, excluídos os feriados.

Como os dados de volume negociado, por ação e no mercado, somente são obtidos para dias úteis, trata-se da única alternativa encontrada. Caso contrário, cálculos como a média, por exemplo, poderiam ser distorcidos caso fosse utilizada a mesma metodologia do Google Trends para os dados de volume obtidos. Assim, os primeiros dados da amostra estão compreendidos na semana de 03.01.2011 a 07.01.2011; já no Google Trends, a primeira semana inicia em 02.01.2011 com término em 08.01.2011. No entanto, independente da métrica de consolidação, foram encontradas 313 semanas (amostra).

Exclusivamente, para as ações da PETROBRAS (PETR3 e PETR4), decidiu-se pela inclusão no estudo do impacto da Operação Lava Jato (Operação) no desempenho dessas ações e, consequentemente, no volume financeiro transacionado. Como meio de pesquisa para avaliar os efeitos dessa investigação, foi incluída uma variável Dummy, onde:

a) Para o período de 01.09.2014 a 29.02.2016 foi atribuído o valor 1 ;

b) Nas semanas anteriores a 01.09.2014 o valor atribuído foi 0 ;

c) Os procedimentos acima foram realizados para 
as ações PETR3 e PETR4 para o período de 02.01.2011 a 25.12.2016; e

d) Nas demais, a Lava Jato recebeu o valor 0 para o período compreendido no intervalo de 313 semanas.

A escolha do período para o qual a variável Dummy assumiria o valor 1 baseou-se em pesquisas realizadas na Internet, de modo a identificar as diversas fases da Operação ${ }^{16}$. Após análise, decidiu-se pelo intervalo do item "a" acima, cujas etapas de investigação sinalizaram um maior envolvimento da PETROBRAS no esquema de corrupção e desvio de dinheiro.

Diante das variáveis apresentadas (explicativas e de controle), tem-se a seguinte regressão final (modelo):

$\operatorname{VOLABN}_{\mathrm{ij}}=\beta_{0}+\beta_{1} \mathrm{MAX}_{\mathrm{iij}}+\beta_{2} \mathrm{MIN}_{\mathrm{ij}}+\beta_{3} \quad \mathrm{RET}_{1 \mathrm{ij}}+\beta_{4}$ $\mathrm{RET}_{2 \mathrm{ij}}+\beta_{5} \mathrm{RET}_{3 \mathrm{ij}}+\beta_{6} \mathrm{RET}_{4 \mathrm{ij}}+\beta_{7} \mathrm{SDVOL}_{\mathrm{ij}}+\beta_{8} \mathrm{EAR}_{\mathrm{ij}}+\beta_{9}$ $\mathrm{TRD}_{\mathrm{ij}}+\beta_{10} \mathrm{LVJ}_{\mathrm{ij}}$

Onde:

$\beta \_1$ MAX_ij+ $\beta \_2$ MIN_ij(Dummy) são apuradas em períodos consecutivos de 52 semanas, entre janeiro de 2010 e dezembro de 2016.

$\beta \_3$ RET_1ij a $\beta \_$(6) RET_4ij representam os retornos durante a semana $-i$, obtidos pela razão entre $o$ último preço e o primeiro preço de fechamento da semana anterior à de observação, excluindo os dividendos.

$\mathrm{SDVOL}_{\mathrm{ij}}$ é desvio padrão das cotações das ações para o intervalo de agosto de 2010 a dezembro de 2016.

$\beta_{8} \mathrm{EAR}_{\mathrm{ij}}$ assume o valor 1 quando do anúncio do pagamento de dividendos por parte das empresas e 0 , caso contrário.

$\beta_{9} \mathrm{TRD}_{\mathrm{ij}}$ é o índice de volume de pesquisa (IVP) semanal realizada no site de busca Google, com variação nos seus valores entre 0 e 100 .

A variável Dummy LVJij assume o valor 1, para o período de setembro de 2014 a fevereiro de 2016, para as ações PETR3 e PETR4, e 0 para as outras observações.

O trabalho apresenta a estrutura de dados em painel e realiza as regressões por meio de Mínimos Quadrados Ordinários (MQO) agrupados (Pooled) e com efeitos fixos de cross-sections. Os dados são apresentados em tabelas para duas categorias de modelos econométricos: agrupados (Pooled), onde para a estimação assume-se que os parâmetros $\alpha$ e $\beta$ são comuns para todas as ações; e com efeito fixo (EF), com a heterogeneidade das ações na parte constante, diferente para cada ativo, alcançando diferenças invariantes no tempo.

Como o número das variáveis que compõem o modelo foi superior ao de cross-sections utilizadas, não foi possível incluir o resultado em painéis com o efeito aleatório ${ }^{17}$. No entanto, os resultados apresentados foram consistentes para os modelos econométricos empregados.

\section{Resultados}

Osmodelos estimados são apresentados nas tabelas 1 e 2. O teste $\mathrm{F}$ indica que os modelos são significativos. Os coeficientes estimados para as variáveis de retorno, a exemplo dos estudos realizado por Huddart, Lang e Yetman (2005), indicam que o volume anormal está associado com o movimento dos preços dos ativos

Tabela 1. Regressões - Efeito Fixo (R\$ em milhares).

Volume anormal - EFEITO FIXO

\begin{tabular}{|c|c|c|c|c|c|c|c|c|c|c|c|c|c|c|}
\hline \multirow[t]{3}{*}{ Regressores } & \multicolumn{8}{|c|}{ IBOVESPA - 9 Ações de alta liquidez } & & & & & & \\
\hline & \multicolumn{3}{|l|}{ Regressão 1} & \multicolumn{2}{|l|}{ Regressão 2} & \multicolumn{3}{|l|}{ Regressão 3} & \multicolumn{3}{|l|}{ Regressão 4} & \multicolumn{3}{|l|}{ Regressão 5} \\
\hline & Coef. & & Std & Coef. & Std & Coef. & & Std & Coef. & & Std & Coef. & & Std \\
\hline Constante & - $3,393.00$ & & $10,356.60$ & $-211,804.90$ & $480,541.50$ & $-247,453.40$ & & $475,732.90$ & $-215,226.70$ & & $473,909.50$ & $-300,304.50$ & & $464,453.00$ \\
\hline MAX & $156,140.40$ & $* \star *$ & $41,623.06$ & $126,899.50$ & $* * * \quad 41,458.57$ & $105,396.80$ & $* * *$ & $40,567.78$ & $101,065.40$ & ** & $41,014.45$ & $113,483.70$ & $* * *$ & $41,002.50$ \\
\hline MIN & - 98,729.24 & ** & $40,033.08$ & $-92,862.23$ & ** $\quad 41,719.35$ & - 90,133.41 & ** & $41,320.86$ & - 99,677.41 & $\star \star$ & $41,005.89$ & $-105,291.30$ & ** & $41,539.52$ \\
\hline RET1 & & & & $80,100.20$ & $231,961.40$ & $59,359.76$ & & $230,830.40$ & $40,366.33$ & & $231,029.60$ & $66,468.69$ & & $226,677.60$ \\
\hline RET3 & & & & $239,738.10$ & $186,045.70$ & $209,763.10$ & & $187,317.70$ & $195,954.90$ & & $187,128.30$ & $223,072.50$ & & $189,856.90$ \\
\hline RET4 & & & & $-179,359.80$ & $195,935.20$ & $-216,916.30$ & & $197,731.90$ & $-227,995.30$ & & $197,734.90$ & - 192,885.90 & & $198,771.70$ \\
\hline VOL & & & & & & $93,266.21$ & $* * *$ & $15,092.88$ & $86,861.78$ & $* * *$ & $15,612.84$ & $64,392.80$ & $* * *$ & $15,817.86$ \\
\hline DIV & & & & & & $55,091.83$ & $* *$ & $26,195.01$ & $52,644.25$ & $* *$ & $26,028.10$ & $57,231.12$ & $* *$ & $26,157.29$ \\
\hline TRENDS & & & & & & & & & $1,046.04$ & $* *$ & 438.11 & 812.61 & * & 449.10 \\
\hline LAVA JATO & & & & & & & & & & & & $264,086.00$ & $* * *$ & $83,114.61$ \\
\hline Cross Section & 9 & & & 9 & & 9 & & & 9 & & & 9 & & \\
\hline R2 - Ajustado & 0.0053 & & & 0.0030 & & 0.0152 & & & 0.01701 & & & 0.02693 & & \\
\hline Estatísitca F & 2.4984 & & & 1.5948 & & 3.6785 & & & 3.8300 & & & 5.2749 & & \\
\hline Prob(Estatística F) & 0.0055 & & & 0.0729 & & 0.0000 & & & - & & & - & & \\
\hline
\end{tabular}


Tabela 2. Regressões - Efeito Agrupado (R\$ em milhares).

Volume anormal - EFEITO AGRUPADO

\begin{tabular}{|c|c|c|c|c|c|c|c|c|c|c|c|c|c|c|c|}
\hline \multirow[t]{3}{*}{ Regressores } & \multicolumn{9}{|c|}{ IBOVESPA - 9 Ações de alta liquidez } & & & & & & \\
\hline & \multicolumn{3}{|l|}{ Regressão 1} & \multicolumn{3}{|l|}{ Regressão 2} & \multicolumn{3}{|l|}{ Regressão 3} & \multicolumn{3}{|l|}{ Regressão 4} & \multicolumn{3}{|l|}{ Regressão 5} \\
\hline & Coef. & & Std & Coef. & & Std & Coef. & & Std & Coef. & & Std & Coef. & & Std \\
\hline Constante & - $\quad 3,421.59$ & & $10,294.02$ & $-212,448.60$ & & $479,087.60$ & $-226,063.20$ & & $476,189.30$ & $-195,278.80$ & & $475,069.30$ & $-255,640.90$ & & $466,512.80$ \\
\hline MAX & $153,065.10$ & $* \star *$ & $41,128.31$ & $125,245.30$ & $* * *$ & $40,790.79$ & $119,457.90$ & $* * *$ & $40,138.09$ & $116,056.20$ & $* * *$ & $40,520.35$ & $126,670.70$ & $* * *$ & $40,258.99$ \\
\hline MIN & - 95,504.77 & ** & $39,386.46$ & - $91,695.09$ & ** & $41,127.34$ & - $98,226.60$ & ** & $41,171.67$ & $-107,225.80$ & $* * *$ & $40,897.51$ & $-112,324.20$ & *** & $41,296.12$ \\
\hline RET1 & & & & $81,032.96$ & & $231,291.00$ & $62,693.26$ & & $230,865.30$ & $45,318.79$ & & $231,293.40$ & $63,657.22$ & & $227,931.60$ \\
\hline RET3 & & & & $239,482.90$ & & $185,793.20$ & $222,743.10$ & & $187,322.60$ & $210,435.20$ & & $187,483.80$ & $230,080.00$ & & $189,415.40$ \\
\hline RET4 & & & & - $179,624.50$ & & $195,778.90$ & $-203,760.60$ & & $197,445.00$ & - 213,722.20 & & $197,770.70$ & $-188,398.70$ & & $198,212.30$ \\
\hline VOL & & & & & & & $55,840.72$ & $* * *$ & $10,203.10$ & $49,125.26$ & $* * *$ & $10,900.80$ & $32,486.67$ & $* * *$ & $10,465.81$ \\
\hline DIV & & & & & & & $51,248.96$ & ** & $25,582.75$ & $49,573.13$ & * & $25,446.87$ & $62,217.83$ & $* *$ & $25,456.25$ \\
\hline TRENDS & & & & & & & & & & 940.06 & $* *$ & 440.07 & 720.86 & & 452.12 \\
\hline LAVA JATO & & & & & & & & & & & & & $222,830.70$ & $* * *$ & $79,420.01$ \\
\hline Obs. & 2,817 & & & 2,817 & & & 2,817 & & & 2,817 & & & 2,817 & & \\
\hline R2 - Ajustado & 0.0079 & & & 0.0057 & & & 0.0134 & & & 0.0149 & & & 0.0236 & & \\
\hline F-estatístico & 12.23 & & & 3.68 & & & 5.73 & & & 5.67 & & & 7.72 & & \\
\hline Prob(F-estatístico) & 0.000005 & & & 0.001209 & & & - & & & - & & & - & & \\
\hline
\end{tabular}

Nota: Nível de Significância: $\left.{ }^{(* \star}\right)$ significativo a $0.01,\left({ }^{* *}\right)$ significativo a 0.05 , e $\left.{ }^{*}\right)$ significarivo a 0.10 .

quando esses ultrapassam os seus mínimos e máximos do intervalo estipulado de 52 semanas. A partir da inclusão dos efeitos da busca por informação (Trends) e da Operação Lava Jato, os resultados se mostraram mais siginificantes, com as variáveis aceitas com nível de p-valor de 0,01, conforme dados da Tabela 1.

Para a Dummy MIN, o comportamento encontrado não segue o movimento esperado de incremento do volume negociado e, ao contrário do previsto, observase uma redução. Esse fenômeno é observado em todas as regressões do modelo proposto, conforme demonstrado nas Tabelas 1 e 2, e pode ser indicativo de uma aversão ao risco por parte do investidor. Assim, quando os preços das ações caem aos seus menores níveis de preço, essa aversão exerceria um desconforto interno que inibiria, por exemplo, a estratégia de compra dessas ações para ganhos adicionais no caso de reversão futura de seus preços.

Esse tipo de decisão, classificada como estratégias contrárias, pode ser considerada como resultado de uma sobre-reação por parte do investidor, onde o desempenho passado dos preços serve de balizador na tomada de decisão. Bondt e Thaler (1985) observaram, empiricamente, para o contexto dos Estados Unidos, no período entre janeiro de 1926 a dezembro 1982, que as carteiras formadas com 35 ações perdedoras superaram o mercado, na média, em $20 \%$, trinta e seis meses após sua formação. As carteiras das ações vencedoras apresentaram desempenho inferior, com ganho aproximadamente $5,0 \%$ menor que o mercado ${ }^{18}$.

Sob essa ótica um desempenho bom (ruim) para determinada ação pode levar a um estado de euforia (desânimo) excessivo, provocando sobre-reação do mercado, onde a prática de compra de ativos com baixo desempenho no passado e a venda de ativos com histórico de bom desempenho é observada. Essa estratégia é baseada na expectativa de reversão dos preços e, segundo Kimura (2003), se ao longo do tempo os preços se ajustarem aos seus valores intrínsecos, a estratégia contrária pode conduzir a retornos acima da média do mercado.

A hipótese principal, que estabelece relação entre um aumento na demanda por certa informação, cujo proxy utilizado neste trabalho foi o Ticker de cada ação, interfere no volume transacionado no mercado também condiz com os coeficientes encontrados. Para tal variável, o modelo econométrico apresentou resultados mais significativos (Tabela 1), embora o $\mathrm{R}^{2}$ ajustado não apresente valores significativos. O Índice de Volume de Pesquisa (IVP) das buscas online no Google capta a atenção dos investidores de varejo, corroborando com Da et al (2011).

Como variável representativa do mercado interno, o termo "Operação Lava Jato" também trouxe contribuição à pesquisa, demostrando que ela influenciou no volume transacionado no mercado. Como demonstrado no item 4.2.4, a exclusão das ações da PETROBRAS do modelo representou queda significativa nos valores dos coeficientes (Dummy MAX) e, consequentemente, no volume agregado do mercado de um modo geral.

A inclusão de variáveis de controle também 
se mostrou útil em trazer robustez ao modelo e, com exceção dos retornos, a volatilidade e o anúncio detiveram seus coeficientes aceitos. Para a volatilidade os coeficientes foram aceitos ao nível de significância de 0,01 nas duas categorias de modelos econométricos: efeito fixo e agrupado.

Os retornos não demonstraram níveis de significância dentro dos intervalos aceitos e, a exemplo da Dummy MIN, os retornos da semana imediatamente anterior à de observação apresentaram valores negativos. Um dos pontos que se destaca com relação aos retornos é que eles não são representativos da entrada de recursos para o investidor, sendo obtidos pela razão entre o preço final e inicial do ativo na semana $i$. Essa constatação pode corroborar o fato de que a atenção do investidor está direcionada para outras variáveis na sua tomada de decisão ou que o período considerado não capta a atenção do investidor. Sugere-se como estudo adicional o cálculo da rentabilidade para intervalos distintos dos aqui apresentados.

Outro ponto a se destacar é que a rentabilidade pode ser mais representativa quando se tem a informação acerca do preço de aquisição dos ativos (compra). Essa informação possibilita validar a hipótese de que os investidores se baseiam em um ponto de referência na avaliação dos resultados obtidos, com valores acima (melhores) do ponto de referência associados como ganhos e valores abaixo (piores) como perdas (KAHNEMAN; TVERSKY, 1979). A pesquisa realizada por Odean (1998), quando analisa a teoria da Disposition Effect, considera que se a verdadeira referência estiver disponível, a evidência estatística seria ainda mais forte para testar a hipótese de que os investidores tendem a manter investimentos com perdas por muito tempo e vender ativos com histórico de ganhos o quanto antes.

\section{Considerações Finais}

Os dados apresentados são consistentes com os estudos de Da et al. (2011). Ou seja, de que o volume de busca na Internet capta a atenção do investidor individual e pode antecipar os preços das ações nas próximas duas semanas. Para a Dummy MIN, todos os coeficientes apresentados no modelo demonstram redução do volume transacionado, diferentemente do que ocorre na Dummy MAX. Esses dados demonstram que, para o mercado doméstico, diferentemente das observações de Bondt e Thaler (1985), para o contexto dos Estados Unidos, não é evidente o uso de estratégias contrárias a partir de uma sobre-reação (Overreaction) por parte do investidor baseada no desempenho passado dos preços das ações.

Os resultados acima não refutam os estudos de Huddart, Lang, Yetman (2005), acerca do fato de que o volume negociado para determinação da ação varia de acordo com a localização do seu preço atual na distribuição em relação ao ano anterior (52 semanas) para diferentes níveis de preços. Mas, para ações de alta liquidez do mercado acionário brasileiro, o volume transacionado não exibe o mesmo sinal quando da ocorrência de seus preços mínimos e máximos. Esse fato pode corroborar os preceitos da teoria da "Disposition Effect" (SHEFRIN; STATMAN, 1985). Sob essa ótica, o efeito da Dummy MIN e o aumento do volume negociado quando o preço corrente das ações encontra-se no nível máximo (Dummy MAX) do intervalo preestabelecido, atesta a hipótese de que os investidores tendem a manter investimentos com perdas por muito tempo e vender ativos com histórico de ganhos o quanto antes.

As variáveis de controle incluídas também se mostraram significativas para o modelo. Quanto à volatilidade, trata-se de medida amplamente utilizada pelos gestores na avaliação dos ativos de suas carteiras. Trata-se de um indicador mais representativo de um comportamento racional na tomada de decisão sobre risco e incerteza. Quanto ao anúncio dos dividendos, mesmo sem a evidenciação de que os valores divulgados pelas empresas eram superiores aos distribuidos anteriormente, é possível concluir por um direcionamento da atenção momentânea do investidor para esse tipo de informação, com possíveis alterações do volume transacionado. Esse efeito não é observado quanto à rentabilidade, talvez pelo fato de que não representa um fluxo de caixa efetivo, cujos coeficientes não são significativos para explicar o volume anormal.

Os resultados obtidos permitem concluir que, ao encontro dos preceitos da Teoria de Perspectiva (KAHNEMAN; TVERSKY, 1979), as escolhas individuais envolvendo resultados monetários e probabilidades conhecidas não se baseiam, exclusivamente, nas premissas da Teoria da Utilidade quanto à racionalidade das escolhas.

O trabalho possui a limitação de analisar somente as ações de empresas de alta liquidez do principal índice de desempenho do mercado de ações. O trabalho não analisa outras ações do índice Bovespa ou de outros índices de mercado de ações. Um desdobramento possível desta pesquisa é estender a análise para as demais empresas cotadas no índice Bovespa e verificar a atenção dos investidores a partir de outros termos de pesquisa no Google além do ticker da ação.

\section{Nota}

1. Hipótese de que os investidores vendem suas ações que geraram perdas ao longo do ano para compensar os ganhos de capital e deduzir nas obrigações tributárias.

2. Tendência dos investidores de vender ativos que geraram ganhos e manter ativos que perderam valor

3.Segundo Stracca (2004), define-se anomalias como traços de comportamento dos agentes de mercado que não podem ser explicados pelo modelo da utilidade esperada ou para indicar um fenômeno que representa uma importante mudança na abordagem convencional da Hipótese de 
Mercados Eficientes (Fama, 1970).

4. Huddart, Lang e Yetman (2005) distinguem os investidores individuais dos institucionais. Por analogia, a distinção seria entre os investidores de varejo e os qualificados (fundações, fundos de pensão e de investimentos, por exemplo)

5. Sua composição é revista a cada quatro meses e o seu "objetivo é ser o indicador do desempenho médio das cotações dos ativos de maior negociabilidade e representatividade" (Disponível em: <http://www. bmfbovespa .com.br pt br/produtos/indices/indices-amplos/indice-bovespaibovespa.htm>. Acesso em: 17/01/2018.).

6. A composição das carteiras do IBOVESPA foi obtida com o auxílio da [B]3, mediante solicitação ao setor de dados históricos, com o respectivo envio da planilha "DECADA10.xls" por e-mail na data de 07/04/2017.

7. Participação relativa das ações da carteira, divulgada para a abertura dos negócios quando do seu início de vigência e sujeita a alterações em função das evoluções dos preços dos papéis que compõem a carteira.

8. Em 22 de março de 2017 ocorreu a fusão da BM\&FBOVESPA S.A. com CETIP S.A. O nome da Empresa foi alterado para Brasil, Bolsa e Balcão [B]3, mas o código de negociação foi mantido em BVMF3.

9. Não é uma medida tão exata, pois no pregão podem ocorrer negociações com preços distintos aos do fechamento.

10. Para o presente estudo: retornos; volatilidade; e anúncio de dividendos.

11. Conforme explicitado no capítulo 3. Figura 2, o preço corrente é apresentado na quinta semana (semana de observação) após decorrido o intervalo de avaliação (range) de 52 semanas.

12. Diante da decisão da empresa pelo pagamento de dividendos aos seus acionistas, conforme previsto na lei das S.A. (Lei n o 6.404, de 15 de dezembro de 1976), os valores podem ser aprovisionados para pagamento em data futura. Huddart, Lang, Yetman (2005) consideraram o valor 1 tanto quando ocorre o anúncio dos dividendos (EARNANN) ou quando dos dados de registro da data dos dividendos.

13. Com base no pressuposto de que o lucro residual da empresa cresça a uma taxa g, supostamente constante ao longo do tempo e compatível com a taxa de crescimento da economia, conforme Modelo de Gordon.

14. O volume de pesquisa reportado pelo Google é, provavelmente, representativo do comportamento de pesquisa na Internet para a população de modo geral Da, Engelberg e Gao (2011).

15. http://www.google.com/trends.

16. Como medida acessória à tomada de decisão acerca da escolha do intervalo, analisou-se o gráfico de desempenho das ações do início da Operação até dezembro de 2016. Observou-se que os picos de altas e baixas no desempenho das ações, representados nos gráficos, compreenderam o período de setembro de 2014 a fevereiro de 2016. Disponível em: http:// www.bmfbovespa.com.br/pt br/servicos/market-data/cotacoes/. Acesso em: 23.02.2018.

17. O modelo de efeito aleatório possui as mesmas suposições do de efeito fixo: o intercepto varia de um indivíduo para outro. A diferença entre os modelos relacionada com o tratamento dado ao intercepto. No modelo de efeito fixo, os interceptos são considerados como fixos, já no aleatório, os interceptos são variáveis aleatórios, onde cada resultado tem a mesma probabilidade de ocorrência.

18. Uma taxa de retorno média aritmética igualmente ponderada em todos os títulos cotados do Centro de Pesquisa em Preços de Ativos serviu como o índice de mercado, Bondt e Thaler (1985).

\section{Referências}

BACHELIER, L. Théorie de la spéculation. Annales Scientifiques de l'École Normale Supérieure, v. 3, n. 17, p. 21-86, 1900.

BANK, M.; LARCH, M.; PETER, G. Google search volume and its influence on liquidity and returns of German stocks. Financial markets and portfolio management, v. 25, n. 3, p. 239-264, 2011
BARBER, B. M., ODEAN, T. Does Online Trading Change Investor Behavior?. Eur Bus Org Law Rev, v. 3, p. 83-128, 2002.

BEAVER, W. H. The Information Content of Annual Earnings Announcements. Journal of Accounting Research, vol. 6, pp. 67-92. 1968

BLANCHARD, O. Speculative bubbles, crashes and rational expectations. Economics Letters, v. 3, n. 4, p. 387-389, 1979.

BONDT, W. F. M.; THALER, R. Does the stock market overreact? The Journal of finance, v. 40, n. 3, p. 793-805, 1985 .

BORGES, E. C. O efeito comportamental na decisão de investimento: o impacto dos preços limite no volume negociado. Dissertação (mestrado). Escola de Administração de Empresas de São Paulo. 2014.

CAMERER, C.; LOVALlO, D. Overconfidence and excess entry: An experimental approach. American economic review, v. 89, n. 1, p. 306-318, 1999.

CASS, D.; SHELL, K. Do Sunspot Matter? The Journal of Political Economy, vol. 91, Issue 2, 193-227. 1983

DA, Z.; ENGELBERG, J; GAO, P. In search of attention. The Journal of Finance, v. 66, n. 5, p. 1461-1499, 2011.

DYL, E. A. Capital Gains Taxation and Year-End Stock Market Behavior. The Journal of Finance, vol. 32, no. 1, pp. 165-175. 1977

FAMA, E. F. The Behavior of Stock-Market Prices. The Journal of Business, vol. 38 , no. 1 , pp. 34-105. 1965.

FAMA, E. F. Efficient capital markets: A review of theory and empirical work. The journal of Finance, v. 25, n. 2, p. 383-417, 1970.

FAMA, E. F. "The Behavior of Stock-Market Prices." The Journal of Business, vol. 38, no. 1, 1965, pp. 34-105.

FERRIS, S. P.; HAUGEN, R. A.; MAKHIJA, A. K. Predicting contemporary volume with historic volume at differential price levels: Evidence supporting the disposition effect. The Journal of Finance, v. 43, n. 3, p. 677-697, 1988.

FISKE, S. T., TAYLOR, S. E. McGraw-Hill series in social psychology. Social cognition (2nd ed.). Mcgraw-Hill Book Company. 1991.

FREDRICKSON, B. L., KAHNEMAN, D. Duration neglect in retrospective evaluations of affective episodes. Journal of Personality and Social Psychology, 65(1), 45-55, 1993

HUDDART, S. J.; LANG, M. H.; YETMAN, M. Psychological factors, stock price paths, and trading volume. SSRN Electronic Journal, 2005.

JOSEPH, K.; WINTOKI, M. B; ZHANG, Z. Forecasting abnormal stock returns and trading volume using investor sentiment: Evidence from online search. International Journal of Forecasting, v. 27, n. 4, p. 1116-1127, 2011.

KAHNEMAN, D. Attention and Effort. Englewood Cliffs, NJ: Prentice-Hall. 1973.

KAHNEMAN, D.; TVERSKY, A. Prospect Theory: An Analysis of Decision under Risk. Econometrica, v. 47, n. 2, p. 263-292, 1979.

KIMURA, H. Aspectos comportamentais associados às reações do mercado de capitais. Revista de Administração de Empresas, v. 2, n. 1, p. 1-14, 2003.

KURZ, M. On the Structure and Diversity of Rational Beliefs, Economic Theory, v. 4, n. 6, p. 877-900, 1994.

KURZ, M., MOTOLESE, M. Endogenous uncertainty and market volatility. Econ Theory, v. 17, p. 497-544, 2001.

LAKONISHOK, J.; SMIDT, S. Volume for winners and losers: Taxation and other motives for stock trading. The Journal of Finance, v. 41, n. 4, p. 951974, 1986.

LIM, K.; LIEW, V. K.; WONG, H. Weak-Form Efficient Market Hypothesis versus Behavioural Finance: A Different Perspective Drawn from the Malaysian Stock Market. International Journal of Management Studies, v. 12, n. 1, p. 1- 28, 2005.

MONDRIA, J.; WU, T.; ZHANG, Y. The determinants of international investment and attention allocation: Using internet search query data. Journal 
of International Economics, v. 82, n. 1, p. 85-95, 2010.

ODEAN, T. Are investors reluctant to realize their losses? The Journal of finance, v. 53, n. 5, p. 1775-1798, 1998.

RIECKEN, F. L. H. W.; SCHACHTER, S. When prophecy fails. University of Minnesota Press, 1956.

RUBIN, A.; RUBIN, E. Informed Investors and the Internet. Journal of Business Finance \& Accounting, v. 37, n. 7-8, p. 841-865, 2010.

SAMUELSON, P. A. Rational Theory of Warrant Pricing". Industrial Management Review, v. 6, n. 2, p. 13-39, 1965.

SHEFRIN, H.; STATMAN, M. The disposition to sell winners too early and ride losers too long: Theory and evidence. The Journal of finance, v. 40, n. 3 , p. 777-790, 1985 .

STARMER, C. Developments in Non-expected Utility Theory: The Hunt for a Descriptive Theory of Choice under Risk. Journal of Economic Literature, v. 38, n. 2 , p. $332-382,2000$

STATMAN, M.; THORLEY, S. Overconfidence, Disposition and Trading Volume. Working paper. Santa Clara University. 1999.

STRACCA, L. Behavioral finance and asset prices: Where do we stand? Journal of Economic Psychology, v. 25, n. 3, p. 373-405, 2004

TUYON, J.; AHMAD, Z. Behavioural finance perspectives on Malaysian stock Market efficiency. Borsa Istanbul Rev., v. 16, n. 1, p. 43-61. 2016

VAN DER SAR, N. L. Behavioral finance: How matters stand. Journal of Economic Psychology, v. 25, n. 3, p. 425-444, 2004

VON NEUMANN, J.; MORGENSTERN, O. Theory of games and economic behavior. 2nd rev. ed. Princeton University Press. 1947.

WYSOCKI, P. D.. Cheap Talk on the Web: The Determinants of Postings on Stock Message Boards (November). University of Michigan Business School Working Paper, n. 98025, 1998. 